\title{
Термофотоэлектрические GaSb-преобразователи излучения инфракрасных селективных эмиттеров
}

\author{
(C) В.П. Хвостиков, С.В. Сорокина, О.А. Хвостикова, М.В. Нахимович, М.З. Шварц
}

Физико-технический институт им. А.Ф. Иофрфе Российской академии наук, 194021 Санкт-Петербург, Россия

E-mail: vlkhv@scell.ioffe.ru

Поступила в Редакцию 31 мая 2021 г.

В окончательной редакции 8 июня 2021 г.

Принята к публикации 8 июня 2021 г.

Исследовались термофотоэлектрические преобразователи на основе $\mathrm{GaSb}$ под излучением селективного $\mathrm{Y}_{2} \mathrm{O}_{3}$-эмиттера мантийного типа, покрытого оксидами редкоземельных элементов $\mathrm{Er}_{2} \mathrm{O}_{3} / \mathrm{Yb}_{2} \mathrm{O}_{3}$. Согласование спектральной чувствительности преобразователей с пиковой длиной волны излучения эмиттера $\lambda=1540$ нм обеспечивает эффективность термофотоэлектрического преобразования > 26\% (0.4 ВТ).

Ключевые слова: термофотоэлектрический преобразователь, $\mathrm{GaSb}$, селективный эмиттер, диффузия.

DOI: $10.21883 /$ FTP.2021.10.51454.9686

\section{1. Введение}

Возможный путь повышения эффективности термофотоэлектрических (ТФЭ) генераторов связан с согласованием ширины запрещенной зоны $\left(E_{g}\right)$ полупроводникового преобразователя с селективной полосой излучения инфракрасного эмиттера. Такой эмиттер должен иметь максимум излучения в диапазоне энергий, близких к $E_{g}$, при сравнительно низких коэффициентах излучения в коротковолновом $\left(h v \gg E_{g}\right)$ и длинноволновом $\left(h v<E_{g}\right)$ диапазонах. По сравнению с абсолютно черным телом при одинаковом количестве подаваемой на излучатель энергии селективный эмиттер будет нагреваться до большей температуры, переизлучая значительную мощность в требуемом диапазоне длин волн. Применяемые в газовых генераторах эмиттеры должны иметь пористую структуру для подачи и горения газа внутри мантийного объема, инициируя нагрев излучателя.

В ТФЭ-системы с GaSb-преобразователями [1-4] могут устанавливаться селективные эмиттеры мантийного типа ${ }^{1}$ с покрытием оксидами редкоземельных элементов $\mathrm{Er}_{2} \mathrm{O}_{3}, \mathrm{Yb}_{2} \mathrm{O}_{3}$ и их комбинациями [5-7], эмиттеры на основе $\mathrm{MoSi}_{2}$ с $\mathrm{Er}_{2} \mathrm{O}_{3}$ [8], $\mathrm{MoSi}_{2} / \mathrm{Er}$ :YAG [9], эмиттеры на фотонных кристаллах [10] и т.д., разработанные для пиковой длины волны $\lambda \approx 1500-1550$ нм. Можно рассматривать и более длинноволновые эмиттеры на основе неструктурированного или микроструктурированного вольфрама с покрытием $\mathrm{HfO}_{2}[11,12]$. В [13] проводилось экспериментальное сравнение 7 типов инфракрасных эмиттеров от Paul Scherrer Institute (включая $\mathrm{Er}_{2} \mathrm{O}_{3}$ - и

\footnotetext{
1 Эмиттер по типу газовой мантии Карла Ауэра фон Вельсбаха, где органическая сетчатая основа покрывается оксидами редкоземельных элементов путем погружения в раствор. В частности, покрытие $\mathrm{Er}_{2} \mathrm{O}_{3}$ получают с использованием водного раствора $\mathrm{Er}\left(\mathrm{NO}_{3}\right)_{3}$, который сначала превращают в гидроксид, а потом путем обжига разлагают в оксид: $\mathrm{Er}\left(\mathrm{NO}_{3}\right)_{3}+3 \mathrm{NaOH} \Rightarrow \operatorname{Er}(\mathrm{OH})_{3}+3 \mathrm{NaNO}_{3}$, $2 \mathrm{Er}(\mathrm{OH})_{3} \Rightarrow \mathrm{Er}_{2} \mathrm{O}_{3}+3 \mathrm{H}_{2} \mathrm{O}[5]$.
}

$\mathrm{Yb}_{2} \mathrm{O}_{3}$-мантии, перфорированные цилиндры из $\mathrm{MoSi}_{2}$, а также тонкостенные пористые цилиндры из $\mathrm{SiC}$ без покрытия или с покрытием оксидами эрбия/иттербия), показавшее их хорошие интегральные (по излучающей способности) и спектральные (селективные) характеристики в диапазоне длин волн 1000-1700 нм, что покрывает диапазон максимальной спектральной фоточувствительности GaSb- [14-16] и Ge-фотопреобразователей [16].

Задача данной работы заключалась в поиске оптимального структурного и технологического решений для $\mathrm{GaSb}$-преобразователей, изготавливаемых по диффузионной технологии в ФТИ им. А.Ф. Иоффе $[14,15,17]$, с целью их фотоэлектрического (по спектральной чувствительности и по кпд) согласования с мантийным эмиттером [13], имеющим покрытие $\mathrm{Er}_{2} \mathrm{O}_{3} / \mathrm{Yb}_{2} \mathrm{O}_{3}$.

\section{2. Селективные эмиттеры для термофотоэлектрических систем}

Излучательная способность эмиттера с покрытием $\mathrm{Er}_{2} \mathrm{O}_{3} / \mathrm{Yb}_{2} \mathrm{O}_{3}$, включающим 15 слоев $\mathrm{Er}_{2} \mathrm{O}_{3}$ и один слой $\mathrm{Yb}_{2} \mathrm{O}_{3}$, приведена на рис. 1 [13]. При температуре $T=1773 \mathrm{~K}$ эмиттер имеет ярко выраженную селективность в диапазоне длин волн излучения 1460-1550 нм (для удобства дальнейших рассуждений данные, представленные на рис. 1, нормированы на интегральную плотность потока излучения $\left.1 \mathrm{BT} / \mathrm{cm}^{2}\right)$. Близкие спектры и аналогичную селективность по длине волны в максимуме излучения дают структуры, состоящие из 10-15 слоев $\mathrm{Er}_{2} \mathrm{O}_{3}$, а также композиция из 15 слоев оксида эрбия и 5 слоев $\mathrm{Yb}_{2} \mathrm{O}_{3}$ [5]. Для сравнения на рис. 1 представлены спектры излучения широкополосных вольфрамовых эмиттеров при температурах $1600-2800 \mathrm{~K}$ с приведенной к $1 \mathrm{BT} / \mathrm{cm}^{2}$ интегральной плотностью потока излучения в плоскости предполагаемого размещения GaSb-преобразователя. 


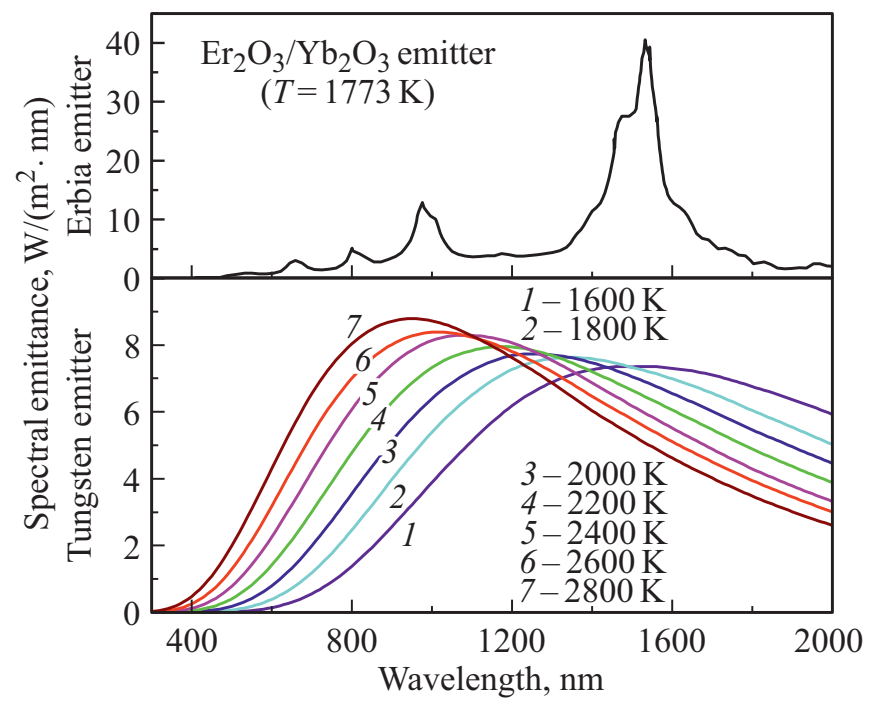

Рис. 1. Излучательная способность мантийного $\mathrm{Er}_{2} \mathrm{O}_{3} / \mathrm{Yb}_{2} \mathrm{O}_{3}$ $(T=1773 \mathrm{~K})$ и вольфрамового $(T=1600-2800 \mathrm{~K})$ эмиттеров при интегральной плотности потока $1 \mathrm{BT} / \mathrm{cm}^{2}$. (Цветной вариант рисунка представлен в электронной версии статьи).

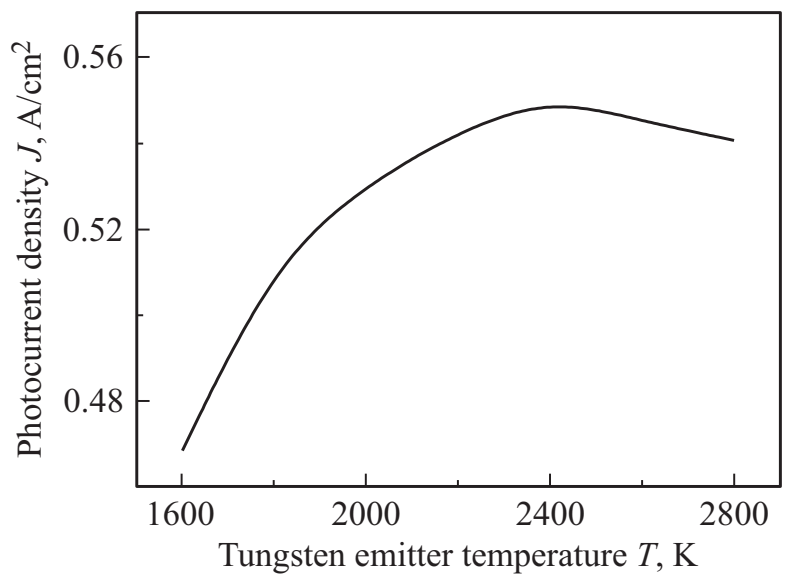

Рис. 2. Зависимость величины плотности фототока GaSbпреобразователя от температуры вольфрамового эмиттера при приведенной к $1 \mathrm{BT} / \mathrm{cm}^{2}$ интегральной плотности потока излучения. Оценки сделаны для GaSb-преобразователя со спектральной чувствительностью, представленной на рис. 3.

Оценки показывают, что при работе с выбранным мантийным $\mathrm{Er}_{2} \mathrm{O}_{3} / \mathrm{Yb}_{2} \mathrm{O}_{3}$-эмиттером $\mathrm{GaSb}$-преобразователь должен иметь плотность фототока на уровне $0.81 \mathrm{~A} / \mathrm{cm}^{2}$. Для широкополосного вольфрамового эмиттера величина плотности фототока GaSb-приемника будет зависеть от температуры излучателя (рис. 2). В силу температурного сдвига спектрального распределения излучения в коротковолновую область наблюдается максимум при 2400 К. Однако, несмотря на достаточно широкий спектр излучения вольфрама и протяженный диапазон спектральной фоточувствительности $\mathrm{GaSb}$-преобразователя, максимальная расчетная плотность фототока не превышает $0.55 \mathrm{~A} / \mathrm{cm}^{2}$ при облученности в $1 \mathrm{BT} / \mathrm{cm}^{2}$.

\section{3. Способ изготовления фотоэлектрических преобразователей}

Структуры GaSb-преобразователей получали диффузией цинка из газовой фазы в подложку $n$-типа проводимости. Применялся двухстадийный вариант изготовления структуры: формирование тонкого p-эмиттера в области фоточувствительной поверхности; заглубление диффузионного $p$-слоя до 1.0-1.5 мкм в подконтактных областях во избежание утечек в $p-n$-переходе или проплавления металлизации. Более подробно такая технология изложена в $[14,15]$. Максимальные значения спектральной чувствительности достигались при малых толщинах диффузионного эмиттера (<0.6 мкм) [15] (см. рис. 3). Антиотражающее покрытие оптимизировалось под минимум отражения в диапазоне длин волн 1500-1600 нм [18]. Малое последовательное сопротивление образцов, работающих при высоких облученностях $\left(15 \mathrm{BT} / \mathrm{cm}^{2}\right.$ и более), обеспечивалось за счет усиленных токоотводящих контактов, формируемых дополнительным высокотемпературным вжиганием золота (до его вплавления в подложку) и повторным напылением/вжиганием контакта.

\section{4. Результаты и обсуждение}

Спектральные зависимости фоточувствительности SR и коэффициента отражения $R$ для $\mathrm{GaSb}$-преобразователя представлены на рис. 3. Оценки $\mathrm{SR}_{\mathrm{int}}$, полученные на основе данных для внутреннего квантового выхода фотоответа $Q_{\text {int }}(\lambda)$ как $\operatorname{SR}_{\text {int }}(\lambda)=q \lambda / h c \cdot Q_{\text {int }}(\lambda)$, оказываются достаточно близкими к предельным, что свидетельствует о высоком качестве фотоактивных слоев, формируемых диффузионным способом.

В конструктивном исполнении $\mathrm{GaSb}$-преобразователей размерами $3.5 \times 3.5,10 \times 10$ мм на лицевой фоточувствительной поверхности предусмотрены контакт-

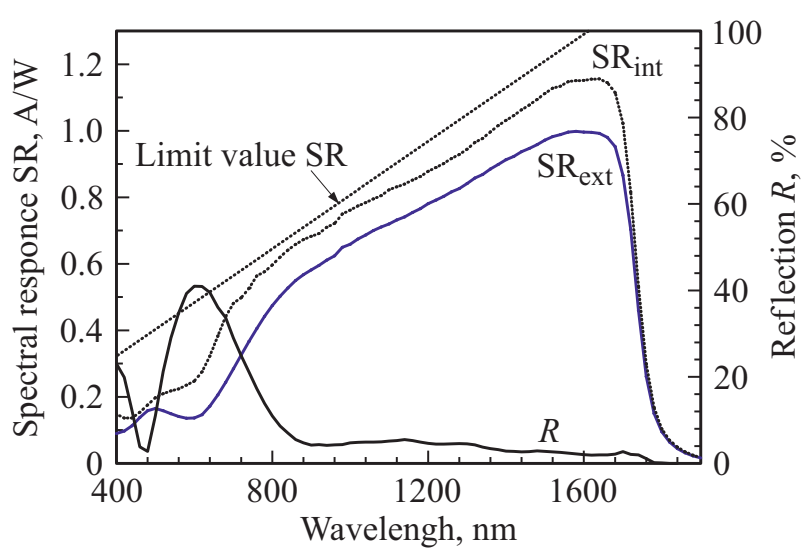

Рис. 3. Спектральная чувствительность (внешняя $\mathrm{SR}_{\mathrm{ext}}$ и внутренняя $\mathrm{SR}_{\mathrm{int}}$ ), а также коэффициент отражения $R$ преобразователя на основе $\mathrm{GaSb}$. 


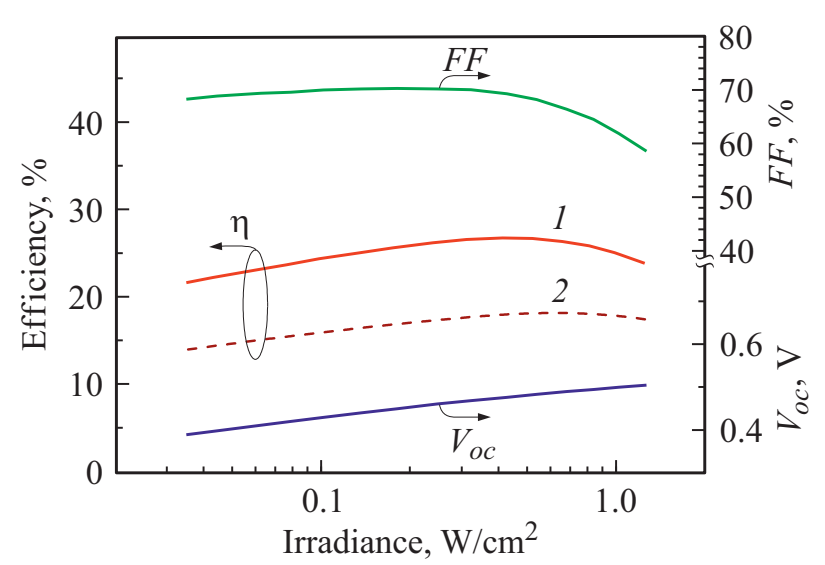

Рис. 4. Зависимость фотоэлектрических параметров GaSbпреобразователя от плотности мощности эмиттера из вольфрама (1) и $\mathrm{Er}_{2} \mathrm{O}_{3} / \mathrm{Yb}_{2} \mathrm{O}_{3}(2)$.

ные полосы, дающие $\sim 8 \%$ затенения с соответствующим увеличением потерь излучения. Таким образом, в реальных приборах с антиотражающим покрытием регистрировались значения спектрального отклика $\quad \mathrm{SR}_{\mathrm{ext}} \geq 0.98 \mathrm{~A} / \mathrm{B}$ для диапазона длин волн $1520-1660$ нм, что соответствует максимальной излучательной эффективности селективного $\mathrm{Er}_{2} \mathrm{O}_{3} / \mathrm{Yb}_{2} \mathrm{O}_{3}$ эмиттера.

Энергетический потенциал разработанных $\mathrm{GaSb}$ преобразователей оценивался в режиме последовательно увеличивающейся мощности излучения от фильтрованного импульсного источника $[19,20]$, что позволяло сохранять изотермический $(298 \mathrm{~K})$ режим работы фотоэлектрического приемника. При моделировании условий облучения, соответствующих спектральному режиму селективного $\mathrm{Er}_{2} \mathrm{O}_{3} / \mathrm{Yb}_{2} \mathrm{O}_{3}$-эмиттера, применялся интерференционный светофильтр, блокирующий коротковолновое (до 1300 нм) и пропускающий длинноволновое (более 1400 нм) излучение. При воспроизведении широкополосного излучения вольфрамового эмиттера спектральная корректировка проводилась в коротковолновом (менее 800-1000 нм) диапазоне длин волн светофильтром из цветного стекла. Длительность плоской части светового импульса составляла 1.2 мс, в течение которой записывалась вольт-амперная характеристика $\mathrm{GaSb}$ преобразователя. Уровень облученности варьировался за счет изменения расстояния от источника излучения до исследуемого образца.

Зависимости фотоэлектрических параметров $\mathrm{GaSb}$ преобразователя (фактора заполнения нагрузочной характеристики $F F$, напряжения холостого хода $V_{o c}$ и эффективности $\eta$ ) от плотности мощности облучения представлены на рис. 4. Для плотности мощности селективного $\mathrm{Er}_{2} \mathrm{O}_{3} / \mathrm{Yb}_{2} \mathrm{O}_{3}$-эмиттера $0.4 \mathrm{BT} / \mathrm{cm}^{2}$ кпд преобразователя приближается к $27 \%$, что соответствует режиму токовой генерации $\sim 350$ мА и выходной электрической мощности $0.11 \mathrm{~B} / \mathrm{cm}^{2}$. Повышение уровня облученности до $1 \mathrm{BT} / \mathrm{cm}^{2}$ приводит к снижению эффективности термофотоэлектрического преобразования до $24 \%$, что следует связывать с ограничениями полупроводниковой структуры (глубина $p-n$-перехода $<0.6$ мкм) и используемой в эксперименте конфигурации контактной сетки



Представленные на рис. 4 данные по кпд $\mathrm{GaSb}$ преобразователя при работе с вольфрамовым эмиттером показывают исключительную значимость взаимного спектрального согласования эмиттера и приемника. Так, из-за распределенной в пределах широкого спектрального диапазона световой энергии наблюдается и меньший генерируемый ток (в силу снижения спектральной чувствительности $\mathrm{GaSb}$ в коротковолновой области, см. рис. 3), и соответствующая этому меньшая эффективность (до 18\%). Ранее для диффузионно-эпитаксиальных образцов кпд также не превышал 16-19\% [4,16] в расчете на преобразование энергии широкополосного вольфрамового излучателя при сравнимых температурах эмиттеров.

\section{5. Заключение}

Представленные в работе оптимизационные решения для $\mathrm{GaSb}$-преобразователя с акцентом на их согласование со спектральными и энергетическими параметрами эмиттеров показывают привлекательность практического применения ТФЭ-систем, использующих энергию горения газового топлива. Это подтверждается и достаточно высокими показателями по достижимым эффективностям GaSb-преобразователей (> 26\%) при их работе со спектрально согласованным мантийным селективным $\mathrm{Er}_{2} \mathrm{O}_{3} / \mathrm{Yb}_{2} \mathrm{O}_{3}$-эмиттером.

Дальнейшее продвижение по шкале эффективностей для ТФЭ-систем и их практическое внедрение будет зависеть от решения ряда во многом противоречивых задач:

- обеспечение высокой температуры при долговременной эксплуатационной надежности селективных эмиттеров;

- увеличение доли „эффективных фотонов“ в общем потоке излучения эмиттеров, повышение энергоотдачи в области пиковой селективности;

- совершенствование структур и конструкций $\mathrm{GaSb}$ преобразователей для повышения кпд при преобразовании излучений высокой плотности $\left(>2-10 \mathrm{BT} / \mathrm{cm}^{2}\right)$;

- снижение стоимости ТФЭ-систем для их эффективной конкуренции по стоимости производимого 1 кВт · ч энергии с традиционными средствами электрогенерации.

\section{Конфликт интересов}

Авторы заявляют, что у них нет конфликта интересов. 


\section{Список литературы}

[1] L. Fraas, J. Avery, L. Minkin. Proc. 9th Annual Int. Energy Conversion Engin. Conf. (San Diego, California, 2011). DOI:10.2514/6.2011-5653

[2] D. Martin, C. Algora, V. Corregidor, A. Datas. ASME J. Solar Energy Engin., 129 (3), 293 (2007). DOI: 10.1115/1.2734567

[3] V.M. Andreev, A.S. Vlasov, V.P. Khvostikov, O.A. Khvostikova, P.Y. Gazaryan, S.V. Sorokina, N.A. Sadchikov. ASME J. Solar Energy Engin., 129 (3), 298 (2007). DOI: $10.1115 / 1.2734576$

[4] A.S. Vlasov, V.P. Khvostikov, S.V. Sorokina, N.S. Potapovich, V.S. Kalinovskiy, E.P. Rakova, V.M. Andreev, A.V. Bobyl, G.F. Tereschenko. Semiconductors, 44 (9), 1244 (2010). DOI: $10.1134 / \mathrm{S} 1063782610090241$

[5] W. Durisch, W. Tobler. Fuel-fired TPV Activities at PSI (Fullspectrum Annual Meeting, 2006, Freiburg, Germany).

[6] E.M. Wong, J.P. Hickey, G.A. Holmquist, P.N. Uppal, C.H. Waldman. AIP Conf. Proc., 358, 278 (1996). DOI: $10.1063 / 1.49693358,278$

[7] A. Gombert. AIP Conf. Proc., 653, 123 (2003). DOI: $10.1063 / 1.1539370$

[8] W.J. Tobler, W. Durisch. Appl. Energy, 85 (5), 371 (2008). DOI:10.1016/j.apenergy.2007.07.015

[9] W.J. Tobler, W. Durisch. High-performance selective Er-oxides emitters on intermetallic $\mathrm{MoSi}_{2}$ substrate (Fullspectrum Annual Meeting, 2007, Malaga, Spain).

[10] V. Stelmakh, W.R. Chan, M. Ghebrebrhan, J. Senkevich, J.D. Joannopoulos, M. Soljačić, I. Celanović. IEEE Trans. Nanotechnology, 15 (2), 303 (2016). DOI: $10.1109 /$ TNANO.2016.2522423

[11] A. Zastrow, B. Bläsi, A. Georg. Fraunhofer ISE Selective Tungsten Emitters for TPV (Fullspectrum Annual Meeting, 2008, Madrid, Spain).

[12] V.M. Andreev, A.S. Vlasov, V.P. Khvostikov, O.A. Khvostikova, P.Y. Gazaryan, N.S. Potapovich. Proc. 23rd Europ. Photovoltaic Solar Energy Conf. (Valencia, Spain, 2008).

[13] W. Durisch, V. Kröni. Fuel-fired TPV Activities at PSI (Fullspectrum Annual Meeting, 2008, Freiburg, Germany).

[14] V.M. Andreev, S.V. Sorokina, N.Kh. Timoshina, V.P. Khvostikov, M.Z. Shvarts. Semiconductors, 43 (5), 668 (2009). DOI: $10.1134 / \mathrm{S} 1063782609050236$

[15] V.P. Khvostikov, S.V. Sorokina, F.Yu. Soldatenkov, N.Kh. Timoshina. Semiconductors, 49 (8), 1079 (2015). DOI: $10.1134 / \mathrm{S} 1063782615080114$

[16] V.P. Khvostikov, O.A. Khvostikova, P.Y. Gazaryan, S.V. Sorokina, N.S. Potapovich, A.V. Malevskaya, N.A. Kaluzhniy, M.Z. Shvarts, V.M. Andreev. ASME J. Solar Energy Engin., 129 (3), 291 (2007). DOI: $10.1115 / 1.2734572$

[17] V.P. Khvostikov, S.V. Sorokina, O.A. Khvostikova, N.K. Timoshina, N.S. Potapovich, B.Y. Ber, D.Y. Kazantsev, V.M. Andreev. Semiconductors, 47 (2), 307 (2013). DOI: $10.1134 / \mathrm{S} 1063782613020139$

[18] V.P. Khvostikov, Ya.V.'Grachev, A.S. Vlasov, O.A. Khvostikova, S.V. Sorokina. J. Power Sources, 501, 229972 (2021). DOI: 10.1016/j.jpowsour.2021.229972

[19] V.D. Rumyantsev, V.M. Andreev, V.R. Larionov, D.A. Malevskiy, M.Z. Shvarts. Proc. Fourth Int. Conf. on Solar Concentrators for the Generation of Electricity or Hydrogen (2007) p. 277.
[20] M.Z. Shvarts, E.D. Filimonov, S.A. Kozhukhovskaia, M.A. Mintairov, N.K. Timoshina, V.M. Andreev. AIP Conf. Proc., 1881, 040006 (2017). DOI: 10.1063/1.5001428

Редактор Л.В. Шаронова

\section{GaSb-based thermophotovoltaic converters for selective IR emitter radiation}

\author{
V.P. Khvostikov, S.V. Sorokina, O.A. Khvostikova, \\ M.V. Nakhimovich, M.Z. Shvarts \\ loffe Institute, \\ Russian Academy of Sciences, \\ 194021 St. Petersburg, Russia
}

Abstract In this study, GaSb-based thermophotovoltaic converters for selective mantle-type $\mathrm{Y}_{2} \mathrm{O}_{3}$-based emitter covered with rare earth oxides $\mathrm{Er}_{2} \mathrm{O}_{3} / \mathrm{Yb}_{2} \mathrm{O}_{3}$ have been investigated. Matching the spectral response of the converters with the peaked emitter radiation wavelength $\lambda=1540 \mathrm{~nm}$ provides a thermophotovoltaic conversion efficiency of more than $26 \%(0.4 \mathrm{~W})$. 